\title{
Insuficiencia renal aguda en adultos críticamente enfermos en dos instituciones hospitalarias.
}

\author{
Acute Kidney failure in critically ill adults in two hospital institutions. \\ Insuficiência renal aguda em adultos criticamente doentes em duas instituições \\ hospitalares
}

\author{
Lina Lozano-Lesmes* \\ Natalia Andrea Quintero-Guzmán** \\ Jenny Lizeth Cuellar-Devia*** \\ Edwin Alberto Torres-García ${ }^{* * * *}$ \\ Samuel Arias-Valencia ${ }^{* * * *}$
}

\section{Resumen}

Objetivo: Cuantificar la incidencia y los factores de exposición relacionados con el desarrollo de la insuficiencia renal aguda en pacientes hospitalizados, en unidades de cuidados intensivos adulto, durante tres meses. Materiales y Métodos: se realizó un estudio epidemiológico de tipo cuantitativo con dos componentes: descriptivo longitudinal y de cohorte histórica; se emplearon los criterios de AKIN; participaron dos instituciones de salud, una de orden privado y otra de orden público. Resultados: Se incluyeron 186 pacientes, con una edad promedio de 56,2 +/- 20,14 años. Se encontró una incidencia del 21,6\% (IC $95 \%$ 17-30) de insuficiencia renal aguda y una tasa de incidencia de 29,2 por cada 100 pacientes por año de exposición. En promedio: la elevación de creatinina fue $0,47 \mathrm{mg} / \mathrm{dL}$ y el gasto urinario de $0,37 \mathrm{cc} /$ $\mathrm{kg} / \mathrm{h}$, los días en ocurrir el evento 3,1 (IC $95 \%$ 2,48-3,74). Igualmente, se halló una relación estadísticamente significativa entre insuficiencia cardíaca aguda 6,84 días (IC $95 \% 4,21-9,48)(\mathrm{p}=0,026)$, posoperatorios 9,82 días (IC $95 \% 8,42-11,2)$ $(\mathrm{p}=0,04)$ y trauma craneoencefálico 4,5 días (IC $95 \% 1,56-7,44)(\mathrm{p}=0,043)$ como causas de ingreso, así como la aparición de insuficiencia renal aguda. Conclusiones: La proporción de incidencia de insuficiencia renal aguda identificada en el presente estudio, es similar a estudios realizados en Colombia; sin embargo, difiere de otros realizados en el exterior. La sepsis fue el diagnóstico con mayor frecuencia entre los pacientes con insuficiencia renal aguda, seguido de la insuficiencia cardíaca aguda. Se encontró significancia estadística en el desarrollo de la insuficiencia renal aguda y la administración de nitroglicerina.

Palabras clave: Enfermedad renal, estudios epidemiológicos, cuidados críticos, adulto.

\section{Autor de correspondencia}

* $\triangle$ Enfermera, Esp. en Epidemiología. Catedrático especialista. Grupo de Cuidado de la SaludUT. Universidad del Tolima. Correo: lilozanoles@ gmail.com. Orcid: iD https://orcid.org/0000-00030860-0476. Bogotá, Colombia

** Enfermera, Esp. en Epidemiología. Profesional contratista en Gobernación del Tolima. Correo: nataquinterog@hotmail.com Orcid: iD https://orcid. org/0000-0002-9384-0302. Ibagué, Colombia.

*** Enfermera, Esp. en Epidemiología. Profesional contratista en Gobernación del Tolima. Correo: jennili21@hotmail.com, Orcid: ID https://orcid. org/0000-0002-9252-5001. Ibagué, Colombia.

**** Médico, Esp. en Epidemiología, especialista en Medicina Interna. Médico Internista, Clínica Medicadiz. Correo: internistapijao@gmail.com. Orcid: (iD https://orcid.org/0000-0002-8895-8242. Ibagué, Colombia.

***** Médico, Mg en Epidemiología. Profesor asociado, Grupo de Epidemiología Universidad de Antioquia. Correo: samuel.arias@udea.edu.co Orcid: (D https://orcid.org/0000-0002-0886-3797. Ibagué, Colombia.

Este es un artículo bajo la licencia CC BY (https://creativecommons.org/ licenses/by/4.0/) @) (1) 
ISSN-PRINT

1794-9831

E-ISSN 2322-7028

Vol. 16 No. 1

Ene - Abr 2019

Cúcuta, Colombia

\begin{abstract}
Objective: To quantify the incidence and factors of exposure related to the development of acute kidney failure in hospitalized patients in the adult intensive care unit during three months. Materials and Methods: An epidemiological study of quantitative type with two components was performed: descriptive longitudinal study and a retrospective cohort study, AKIN criteria were used, two health care institutions (one private and one public) participated. Results: 186 patients were included with an average age of $56.2+/-20,14$ years. An incidence of $21.6 \%$ was found (IC $95 \% 17-30$ ) of acute kidney failure and a rate of incidence of 29.2 for every 100 patients / year of exposure. On average the creatinine elevation was of $0.47 \mathrm{mg} / \mathrm{dL}$ and the urine output was of $0.37 \mathrm{cc} / \mathrm{kg} / \mathrm{h}$, days of event occurrence 3.1 (IC $95 \%$ 2.48-3.74). A significant statistical relationship was found between acute heart failure 6.84 days (IC $95 \%$ 4.21-9.48) ( $\mathrm{p}=0.026$ ), post-operatives 9.82 days (IC $95 \% 8.42-11.2$ ) $(\mathrm{p}=0.04)$ and traumatic brain injury 4.5 days (IC $95 \% 1.56-7.44)(\mathrm{p}=0.043)$, as causes of admission and appearance of acute kidney failure. Conclusions: The incidence proportion of acute kidney failure identified in the present study, is similar to other studies performed in Colombia, however, it differs from studies performed in other countries. Sepsis was the most frequent diagnosis among patients with acute kidney failure, followed by acute heart failure. Statistical significance was found in the development of acute kidney failure and the intake of nitroglycerin.
\end{abstract}

Keywords: Kidney failure, Epidemiological studies, Critical care, Adult.

\title{
Resumo
}

Objetivo: Quantificar a incidência e os fatores de exposição relacionados com o desenvolvimento da Insuficiência Renal Aguda (IRA) em pacientes adultos hospitalizados em unidades de terapia intensiva durante três meses. Materiais e Métodos: realizou-se um estudo epidemiológico de tipo quantitativo com dois componentes: descritivo longitudinal e de coorte histórica, empregaram-se os critérios de AKIN (Acute Kidney Injury Network), participaram duas instituições de saúde, uma de ordem privado e outra de ordem pública. Resultados: Incluíram-se 186 pacientes, com uma idade média de 56,2 $\pm 20,14$ anos. Encontrou-se uma incidência do 21,6 \% (IC $95 \%$ 17-30) de insuficiência renal aguda e uma taxa de incidência de 29,2 por cada 100 pacientes ano exposição. Em média a elevação de creatinina foi de $0,47 \mathrm{mg} / \mathrm{dL}$ e o gasto urinário de $0,37 \mathrm{cc} / \mathrm{kg} / \mathrm{h}$, os dias em ocorrer o evento 3,1 (IC $95 \%$ 2,48-3,74). Encontrou-se relação estatisticamente significativa entre insuficiência cardíaca aguda (ICA) 6,84 dias (IC $95 \% 4,21-9,48)(\mathrm{p}=0,026)$, pós-operatórios 9,82 dias (IC $95 \% 8,42-11,2)(\mathrm{p}=0,04)$ e traumatismo cranioencefálico (TCE) 4,5 dias (IC $95 \%$ 1,56-7,44) $(\mathrm{p}=0,043)$ como causas de ingresso e a aparição de insuficiência renal aguda. Conclusões: A proporção de incidência de insuficiência renal aguda identificada no presente estudo, é similar a estudos realizados na Colômbia, no entanto, difere de outros realizados no exterior. A sepse foi o diagnóstico com maior frequência entre os pacientes com insuficiência renal aguda, seguido da insuficiência cardíaca aguda. Obteve-se significância estadística no desenvolvimento da insuficiência renal aguda e a administração de nitroglicerina.

Palavras-chave: Adulto, Cuidados Críticos, Doença renal, Estudos Epidemiológicos.

\section{Introducción}

La Insuficiencia Renal Aguda (IRA) se define como el deterioro abrupto de la función renal, traducida en la caída de la filtración a través de los glomérulos, elevación de creatinina y BUN con reducción o no del volumen urinario $(1,2)$.

La mayor complicación de la lesión renal aguda es la enfermedad renal crónica. Situación bastante alarmante, pues ésta patología, de acuerdo a varios au- tores, genera aumento de la carga de la enfermedad tanto para el sistema de salud (3-6), como para los pacientes, las familias y cuidadores. También contribuye a la disminución de la calidad de vida de los pacientes, pues la enfermedad renal crónica como complicación de la IRA, requiere terapia de remplazo renal o la inclusión a listas de espera para trasplante renal, según López-Soracipa et al. (7).

En China, Zhou et al. (8), encontraron una proporción de incidencia de $34,1 \%$ con una mortalidad del 
54,4 \%. En Cuba, Capote Leyva et al. (9), reportan una incidencia de 100 por millón de habitantes y en pacientes críticos la incidencia varía entre un 7-30\%, en un estudio retrospectivo y descriptivo que incluyó a pacientes que ingresaron a unidades de cuidado intensivo se encontró una letalidad de $61,1 \%$.

En Colombia Moreno et al. (10) realizaron un estudio en el 2006 en una Unidad de Cuidado Intensivo (UCI) encontrando una incidencia del $22 \%$ de falla renal aguda; las comorbilidades fueron: diabetes, hipertensión arterial, enfermedad pulmonar obstructiva crónica, noradrenalina más dobutamina, provocando una mortalidad del 13,4 \%.

Según a Khwaja (11) las causas de lesión renal aguda suelen variar de acuerdo al manejo médico proporcionado a los pacientes, pero es frecuente que la causa más importante sea la necrosis tubular aguda, ya sea isquémica $54 \%$, tóxica $8 \%$ o mixta $21 \%$. La segunda etiología más frecuente es la lesión renal aguda pre-renal que se observa en el $12 \%$ de los casos, y se debe al bajo volumen intravascular y al estado hemodinámico del paciente; el 35-50 \% de las necrosis tubulares agudas son secundarias a la sepsis. En cuanto al tipo de la sepsis, según Cortés et al. (12) hasta el $19 \%, 23 \%$ y $51 \%$ de los pacientes con sepsis, sepsis severa o choque séptico, respectivamente, desarrollan necrosis tubular aguda. Además, la necrosis tubular aguda es extremadamente frecuente en el contexto de falla multiorgánica.

La segunda causa es post quirúrgica y la tercera es medicamentosa, especialmente relacionada con el uso de medios de contraste. Otros fármacos relacionados con el desarrollo de necrosis tubular aguda son antibacterianos como los: aminoglucósidos o la vancomicina, antimicóticos como la anfotericina $\mathrm{B}$ y múltiples fármacos antineoplásicos (12-14).

Vallabhajosyula et al. (15) demostraron la IRA como factor de riesgo en sepsis independientemente de la mortalidad, así como lo es la lesión cardiovascular. También identificaron otros factores de riesgo que están relacionados con el ingreso del paciente al servicio de salud por lesión renal aguda como: la edad por encima de 65 años, sepsis como causa de admisión a la UCI, falla cardiovascular, cirrosis hepática, falla respiratoria, falla cardíaca crónica, síndromes linfoproliferativos, $\mathrm{pH}$ menor de 7,3. Por otra parte Kellum (16) identificó que la nefropatía por medio de contraste se relaciona con: la función renal de base, diabetes mellitus, falla cardíaca, depleción de volumen intravascular, uso concomitante de medicamentos nefrotóxicos, volumen y medio de contraste hiperosmolar, inestabilidad hemodinámica $\mathrm{y}$ anemia.

La IRA es una de las complicaciones más frecuentes en la UCI; según Kashani et al. (17) aquellos pacientes que la padecen están expuestos a un riesgo mayor de muerte antes del alta hospitalaria y los supervivientes tienen un riesgo significativo a corto y largo plazo, por tanto, "la identificación de los factores de riesgo y su manejo temprano y oportuno, implicarían optimización de recursos, mejoría en la calidad de la atención y disminución de complicaciones y morbimortalidad". (10).

Dado que la lesión renal aguda es una complicación frecuente en la UCI, si no se identifica a tiempo es muy difícil tratarla, por lo que, lo más importante es su prevención; por tanto, se debe conocer el comportamiento que puede ayudar a dirigir el respectivo tratamiento y así evitar el riesgo en los pacientes, obteniendo mejores resultados clínicos para convertir en más eficiente el sistema de salud, en este grupo poblacional.

\section{Objetivos}

\section{Objetivo general}

Medir la incidencia y los factores de exposición relacionados con el desarrollo de la insuficiencia renal aguda en pacientes hospitalizados en las unidades de cuidados intensivos para adulto de dos instituciones Hospitalarias, en el periodo comprendido entre septiembre y noviembre de 2012.

\section{Objetivos específicos}

- Describir las características demográficas, sexo, edad y clínicas relacionadas con: diagnóstico de ingreso, antecedentes patológicos y quirúrgicos e intervenciones médicas realizadas en la hospitalización, en la unidad de cuidados intensivos de los pacientes

- Determinar la incidencia de la insuficiencia renal aguda de los pacientes en las unidades de cuidados intensivos, por medio de los criterios de AKIN
E-ISSN 2322-7028

Vol. 16 No. 1

Ene - Abr 2019

Cúcuta, Colombia 
ISSN-PRINT

1794-9831

E-ISSN 2322-7028

Vol. 16 No. 1

Ene - Abr 2019

Cúcuta, Colombia
- Cuantificar el tiempo desde el ingreso de los pacientes a las unidades de cuidado intensivo hasta la aparición de la insuficiencia renal aguda.

- Establecer si existe relación entre los antecedentes patológicos personales y el diagnóstico de ingreso, con el desarrollo de insuficiencia renal aguda en los pacientes de las unidades de cuidado intensivo

- Describir el tipo de intervenciones farmacológicas realizadas a los pacientes hospitalizados en la unidad de cuidados intensivos y la relación entre estas y la aparición de la insuficiencia renal aguda

\section{Materiales Y Métodos}

Se trata de un estudio epidemiológico de tipo cuantitativo con dos componentes: el descriptivo longitudinal, para la caracterización clínica y el tiempo al evento y de cohorte para establecer la asociación entre exposiciones de antecedentes, clínicas y terapéuticas con el riesgo de IRA. Todo esto para conocer el comportamiento de la insuficiencia renal aguda y los factores relacionados con el desarrollo de ésta patología.

Participaron dos instituciones de salud de tercer nivel de la ciudad de Ibagué, en el departamento del Tolima:una Empresa social del Estado, denominada Hospital y una empresa privada, designada como Clínica.

La población de estudio corresponde a todos los pacientes adultos que ingresaron a la UCI. La muestra fue la misma población, dadas las características y alcances del estudio. Se tomó la base de datos de pacientes que ingresaron a las tres unidades de cuidado intensivo desde el 1 de septiembre hasta el 30 de noviembre de 2012; se incluyeron pacientes mayores de 18 años, sin antecedentes de enfermedad renal, con función renal normal al ingreso. También se realizó el seguimiento a los valores de creatinina diarios, al gasto urinario y a los criterios de AKIN. Una vez que el paciente cumpliera con dichos criterios, se midieron variables como: medicamentos a los que estuvo expuesto, el diagnóstico en el momento de presentar IRA y además se llevó a cabo $r$ un análisis de tiempo al evento.
Los criterios de exclusión que se tuvieron en cuenta hacen referencia a pacientes mayores de 18 años que fueron remitidos de otras unidades de cuidados intensivos adultos y con valor de creatinina alterada. Los valores límites para la creatinina dependieron de los establecidos por el laboratorio de cada institución, de tal manera que para el Hospital, el rango de los niveles de creatinina fue de 0,5 a 1,0 $\mathrm{mg} / \mathrm{dl}$ y para la Clínica 0,6 a $1,2 \mathrm{mg} / \mathrm{dl}$.

Para identificar la IRA se emplearon los criterios de AKIN definidos como incremento mayor de $0,3 \mathrm{mg} /$ dl de la creatinina basal o incremento de más de 1,5 veces en la creatinina sérica basal o disminución del volumen urinario por debajo de $0,5 \mathrm{ml} / \mathrm{kg} / \mathrm{h}$ durante más de 6 horas, según Mehta et al. (18).

Para lograr los objetivos propuestos se diseñó un instrumento de recolección de datos, divido en tres secciones: la primera con las características demográficas sexo y edad, los datos de ingreso como causa de ingreso, antecedentes patológicos, fecha de ingreso, lugar de remisión y valor de creatinina al ingreso. Al completar la primera sección se decidía excluir o no al paciente. La segunda medía las variables relacionadas al momento de presentar IRA, como valor de creatinina, gasto urinario, fecha de alteración de estos valores y diagnóstico. La tercera determinó las variables relacionadas con las intervenciones terapéuticas a las que estuvieron expuestos todos los pacientes incluidos en el estudio, además de la información del egreso del paciente que incluye la fecha y el lugar de egreso.

Se aplicó una prueba piloto a 10 historias clínicas, 5 en cada institución, lo que permitió detectar falencias en el instrumento y en la fuente de información. Una vez realizada esta prueba piloto, se ajustó el instrumento y se inició la recolección de la información.

La obtención de los datos se obtuvo a través de la historia clínica de los pacientes incluidos en el estudio y de los libros de ingreso y egreso las unidades de cuidado intensivo. Una vez recolectados los datos fueron verificados y dispuestos en una base de datos. Para el análisis estadístico se utilizó el programa SPSS Statistics versión 19.0 y Epidat versión 3.1.

De igual manera, se realizó un análisis univariado para caracterizar a los pacientes del estudio y las 
intervenciones farmacológicas a las que estuvieron expuestos, a través de medidas de tendencia central como: desviación estándar, coeficiente de variación, cuartiles, mínimo, máximo, frecuencia absoluta y relativa, proporciones, razones y análisis de subgrupos con indicadores demográficas por género e indicadores de exposición por sexo y edad.

Asimismo, para cuantificar el tiempo desde el ingreso de los pacientes a las unidades de cuidado intensivo hasta la aparición de la IRA se realizó un análisis de supervivencia con el método Kaplan-Meier.

Este estudio cuenta con la aprobación del Comité de ética de las dos instituciones de salud, conforme a la resolución 8430 de 1993, teniendo en cuenta el diseño metodológico presenta una clasificación de investigación sin riesgo.

\section{Resultados}

De los 339 pacientes que ingresaron a las unidades de cuidado intensivo, no se incluyeron 153 pacientes, de los cuales el 93,5 \% ingresaron con función renal alterada y el 7,8 \% fueron remitidos de otras unidades de cuidado intensivo. El $23 \%$ de los pacientes que presentaron alteración de la función renal al ingreso, tenía enfermedad renal previa. El valor de creatinina presentado por los pacientes excluidos en promedio fue de $2,78 \mathrm{mg} / \mathrm{dl}$. El $50 \%$ tenía creatinina de 1,57 $\mathrm{mg} / \mathrm{dl}$ con un mínimo de $0,5 \mathrm{mg} / \mathrm{dl}$ y un máximo valor de $19,1 \mathrm{mg} / \mathrm{dl}$.

Se estudiaron 186 pacientes de las tres unidades de cuidado intensivo, el 52,7 \% eran mujeres y el 47,3\% hombres. La edad promedio fue 56,26 años con una desviación estándar de 20,14 años. La hipertensión arterial como antecedente fue la más frecuente con $40,9 \%$ seguida de antecedente de cirugía menor. El evento coronario $25,8 \%$, posoperatorios $23,2 \%$, trauma craneoencefálico $14 \%$, la sepsis $13,5 \%$ y la insuficiencia cardíaca aguda $12,7 \%$ fueron las principales causas de ingreso de los pacientes del estudio. Ver tabla 1.

Tabla 1. Características clínicas de pacientes incluidas en el estudio.

\begin{tabular}{llcc}
\hline Características clínicas & $\mathrm{n}=186$ & $\%$ \\
\hline & Hipertensión arterial & 76 & 40,9 \\
& Otros & 65 & 34,9 \\
& Cirugía Menor & 29 & 15,6 \\
& Cirugía mayor & 18 & 9,7 \\
Antecedentes & Enfermedad Pulmonar obstruc- & 17 & 9,1 \\
personales & tiva crónica & 16 & 8,6 \\
& Diabetes Mellitus & 13 & 7 \\
& Hipotiroidismo & 12 & 6,5 \\
& Insuficiencia cardíaca conges- & 10 & 5,4 \\
& tiva & 59 & 31,9 \\
& Síndrome coronario & 48 & 25.8 \\
& Otros & 43 & 23,2 \\
& Evento Coronario & 26 & 14 \\
& Posoperatorios & 25 & 13,5 \\
& Trauma craneoencefálico & 24 & 12,9 \\
& Sepsis & 13 & 7 \\
& Insuficiencia cardíaca aguda & 10 & 5,4 \\
Diagnóstico de & Arritmias & 10 & 5,4 \\
\cline { 2 - 4 } & Traumas & 9 & 4,8 \\
& Hemorragias cerebrales & 8 & 4,3 \\
& Dengue & 8 & 4,3 \\
& Evento cerebrovascular & 7 & 3,8 \\
& Crisis hipertensiva & 5 & 2,7 \\
\hline & Enfermedad Pulmonar obstruc- & &
\end{tabular}

Fuente: Datos de la investigación 
ISSN-PRINT

1794-9831

E-ISSN 2322-7028

Vol. 16 No. 1

Ene - Abr 2019

Cúcuta, Colombia
En cuanto a la aparición de IRA encontramos que por cada 100 pacientes año exposición, 29,2 desarrollaron el evento y la incidencia de insuficiencia renal aguda en los pacientes del estudio fue de 21,6\% (IC $95 \%$ 17-30). En la UCI de la Clínica la incidencia de insuficiencia renal aguda fue de $21,9 \%$ y en las unidades de cuidado intensivo del Hospital fue 22,2 \% (IC95 0,571,69). La incidencia de IRA fue mayor en los hombres con $54,4 \%$ comparado con la incidencia en mujeres $46,3 \%$ (IC $95 \%$ 59-100); en los mayores de 63 años con un $61,3 \%$ y en los menores de 62 se presentó una incidencia de falla renal aguda en 36,5\% (IC $95 \%$ 0,39- 0,91).

De los casos de IRA el 36,6 \% fue identificado por disminución de gasto urinario $<0,5 \mathrm{cc} / \mathrm{kg} / \mathrm{h}$, el 53,7 $\%$ por elevación de la creatina $0,3 \mathrm{mg} / \mathrm{dl}$ respecto a la basal o superar el valor máximo definido por el la- boratorio. El 9,8 \% presentó los dos criterios. El promedio de elevación de creatinina en los pacientes que presentaron insuficiencia renal aguda fue $0,47 \mathrm{mg} / \mathrm{dl}$ con una variabilidad global con respecto al promedio de más o menos $0,44 \mathrm{mg} / \mathrm{dl}$. El promedio del gasto urinario de los pacientes con insuficiencia renal aguda fue $0,37 \mathrm{cc} / \mathrm{kg} / \mathrm{h}$.

Por otra parte, el promedio en días en el que los pacientes que ingresan a las unidades de cuidado intensivo tardan en desarrollar de insuficiencia renal aguda fue de 3,1 (IC $95 \%$ 2,48-3,74). La probabilidad de presentar insuficiencia renal aguda a los 3 días en los pacientes que ingresaron a las unidades de cuidado intensivo del Hospital y la Clínica fue del $82 \%$ y a los 6 días del $70 \%$ (Figura 1).

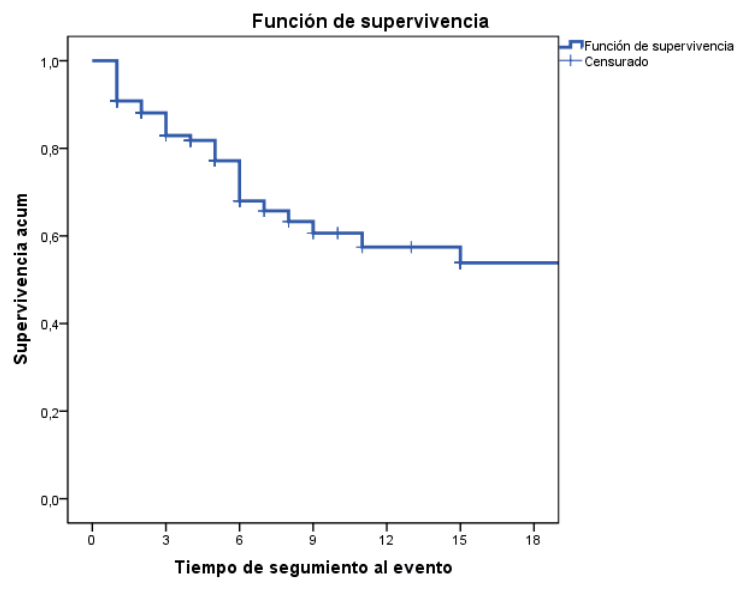

Figura 1. Tiempo al evento de los pacientes desde el ingreso hasta el momento de presentar IRA.

Fuente: Autor

En cuanto a los diagnósticos de ingreso en los pacientes que presentaron insuficiencia renal aguda se identificaron: insuficiencia cardíaca aguda, posoperatorios, trauma craneoencefálico, evento coronario agudo y sepsis. Ver tabla 2 . 
Tabla 2. Media y mediana del tiempo de supervivencia de los pacientes que ingresaron a las Unidades de cuidado intensivo y presentaron insuficiencia renal aguda.

\begin{tabular}{ccccc}
\hline Diagnóstico de ingreso & & Categoría & Media & IC 95\% \\
\hline \multirow{2}{*}{ Insuficiencia cardíaca aguda } & - & $\mathrm{Si}$ & 6,84 & $4,21-9,48$ \\
& - & $\mathrm{No}$ & 33,46 & $27,1-39,7$ \\
\multirow{2}{*}{ Posoperatorios } & - & $\mathrm{Si}$ & 9,82 & $8,42-11,2$ \\
& - & $\mathrm{No}$ & 45,83 & $39,8-51,8$ \\
\multirow{2}{*}{ Trauma craneoencefálico } & - & $\mathrm{Si}$ & 4,50 & $1,56-7,44$ \\
\multirow{2}{*}{ Evento coronario agudo } & - & $\mathrm{No}$ & 31,89 & $25,5-38,2$ \\
& - & $\mathrm{Si}$ & 10,48 & $7,12-13,8$ \\
& - & $\mathrm{No}$ & 30,92 & $24,1-37,7$ \\
Sepsis & - & $\mathrm{Si}$ & 8,29 & $5,22-11,3$ \\
& - & $\mathrm{No}$ & 32,93 & $26,2-39,5$ \\
\hline
\end{tabular}

Fuente: Datos de Investigación

El tiempo promedio en desarrollar insuficiencia renal aguda en pacientes de las unidades de cuidado intensivo con diagnóstico de insuficiencia cardíaca aguda fue de 6,84 días (IC 95\% 4,21-9,48), el $50 \%$ de ellos presentaron el evento a los 6 días o menos y el $75 \%$ a los 4 días o menos.

Se observó que a los tres días el $78 \%$ de los pacientes con insuficiencia cardíaca aguda presentaron insuficiencia renal aguda y a los 6 días el $42 \%$. A los tres días, los pacientes con diagnóstico de posoperatorios, desarrollaron insuficiencia renal aguda hasta en un 96 $\%$, luego de esta fecha presentaron en un $85 \%$ hasta finalizar el tiempo de seguimiento. También, se percibió que a los 3 días el $58 \%$ de los pacientes que tenían trauma craneoencefálico como diagnóstico, presentaron insuficiencia renal aguda, situación clínica que mantiene igual comportamiento hasta los seis días.

En cuanto a los diagnósticos al momento de presentarse insuficiencia renal aguda, se evidenció que el más frecuente fue la sepsis, siendo de mayor prevalencia en hombres en un 9,1\% con respecto al 8,1\% de las mujeres; también prevalecieron otros diagnósticos como: tromboembolismo pulmonar, desequilibrio electrolítico, eventos cerebrovasculares, dengue, los cuales de forma individual no son tan frecuentes. El segundo diagnóstico de mayor frecuencia fue la insuficiencia cardíaca aguda en un 5,4\%, presentándose en los hombres en un $6,8 \%$ y en las mujeres en el $4 \%$; le siguen los eventos coronarios agudos en un $4,3 \%$ distribuyéndose en un 6,8\% en hombres frente al $2 \%$ en las mujeres.

De acuerdo a la presentación de la insuficiencia renal aguda en las dos instituciones se encontró una distribución heterogénea de los diagnósticos actuales (Ver tabla 3). Con respecto a las intervenciones farmacológicas se descubrió que $20 \%$ de los pacientes que ingresaron a las unidades de cuidado intensivo recibió noradrenalina, el $16,7 \%$ estuvo expuesto a medios de contraste, $9,2 \%$ a nitroglicerina, $8,6 \%$ a nitroprusiato, $7,0 \%$ a vancomicina y el mismo porcentaje a dopamina. La vasopresina, amikacina y anfotericina $\mathrm{B}$ fueron las intervenciones con una frecuencia menor de $4 \%$. 
ISSN-PRINT

1794-9831

E-ISSN 2322-7028

Vol. 16 No. 1

Ene - Abr 2019

Cúcuta, Colombia

Tabla 3. Diagnósticos e intervenciones farmacológicas en los pacientes con IRA de acuerdo a las instituciones.

\begin{tabular}{ccccccccc}
\hline & \multicolumn{2}{c}{ Sepsis } & \multicolumn{2}{c}{$\begin{array}{c}\text { Insuficiencia Cardíaca } \\
\text { aguda }\end{array}$} & \multicolumn{2}{c}{$\begin{array}{c}\text { Evento } \\
\text { coronario }\end{array}$} & \multicolumn{2}{c}{ Posoperatorios } \\
\cline { 2 - 8 } Ucis & $\mathrm{n}=41$ & $\%$ & $\mathrm{n}=41$ & $\%$ & $\mathrm{n}=41$ & $\%$ & $\mathrm{n}=41$ & $\%$ \\
Clínica & 7 & 17 & 10 & 24.3 & 6 & 14.6 & 2 & 4.8 \\
Hospital & 9 & 22 & 0 & 0 & 2 & 4.8 & 4 & 9.7 \\
& Noradrenalina & Nitroglicerina & Dopamina & Vancomicina \\
& $\mathrm{n}=41$ & $\%$ & $\mathrm{n}=41$ & $\%$ & $\mathrm{n}=41$ & $\%$ & $\mathrm{n}=41$ & $\%$ \\
Clínica & 11 & 26.8 & 7 & 17 & 6 & 14.6 & 3 & 7.3 \\
Hospital & 05 & 12.1 & 1 & 2.4 & 0 & 0 & 3 & 7.3 \\
\hline
\end{tabular}

Fuente: Datos de la investigación

En cuanto a las intervenciones farmacológicas y su relación con la insuficiencia renal aguda, se encontró que el $47 \%$ de los pacientes expuestos a nitroglicerina presentó el evento y el $18,7 \%$ de los que recibieron Nitroprusiato desarrollaron IRA. En cuanto a los pacientes a los que se les administró noradrenalina el 43,2 \% desarrolló IRA y de todos los expuestos a medios de contraste el 9,6\% presentaron la situación clínica. Igualmente, se observó diferencia en la distribución de la insuficiencia renal aguda de acuerdo a la exposición de las intervenciones farmacológicas en los pacientes de las diferentes instituciones. El promedio que desarrolló insuficiencia renal aguda y a quienes se les administró nitroglicerina fue de 6,79 días (IC 95 4,83-8,76). El 25 \% presentó el evento a los 9 días o menos y tanto el 50 como el $75 \%$ presentaron IRA a los 6 días o menos. También, se percibió que a los tres días el $82 \%$ de los pacientes a quienes se les administró nitroglicerina intravenosa, presentan IRA y éste comportamiento se conserva hasta el sexto día, en donde se desarrolló la situación clínica en un $50 \%$ (Ver tabla 4).

Tabla 4. Media del tiempo de supervivencia de los pacientes expuestos a intervenciones farmacológicas y la IRA.

\begin{tabular}{ccccc}
\hline Intervenciones farmacológicas & Categoría & Media & IC 95\% & Valor de P \\
\hline \multirow{2}{*}{ Nitroglicerina } & $\mathrm{Si}$ & 6,79 & $4,83-8,76$ & 0,034 \\
& $\mathrm{No}$ & 35,31 & $29,3-41,2$ & \\
Medios de contraste & $\mathrm{Si}$ & 12,99 & $10,7-15,2$ & 0,071 \\
& $\mathrm{No}$ & 29,10 & $22,1-36,0$ & \\
Noradrenalina & $\mathrm{Si}$ & 9,35 & $7,33-11,3$ & 0,128 \\
& $\mathrm{No}$ & 33,1 & $24,7-41,6$ & \multirow{2}{*}{0,547} \\
\hline \multirow{2}{*}{ Nitroprusiato } & $\mathrm{Si}$ & 11,25 & $7,62-14,6$ & \\
\hline
\end{tabular}

Fuente: Datos de la investigación

\section{Discusión}

La insuficiencia renal aguda es una patología que ha sido poco estudiada en nuestro país y se desconoce su comportamiento en el departamento del Tolima. En el estudio se encontró una población adulta en donde el $75 \%$ de los pacientes tenía 74 años o menos, constituyéndose en un factor importante para la presencia de enfermedades crónicas como la insuficiencia cardíaca.
Dentro de los antecedentes patológicos se hallaron las enfermedades crónicas como hipertensión, EPOC y diabetes con un 58,6\% y los antecedentes quirúrgicos representaron un $25 \%$.

Debe señalarse, que coinciden las causas de ingreso a las unidades de cuidado intensivo con lo presentado por la Secretaria de Salud del Tolima en el Análisis de situación en salud (19), dado que el evento coronario fue el 
principal diagnóstico al ingreso de los pacientes estudiados con una frecuencia del $25,8 \%$, presentándose con mayor frecuencia en los pacientes con 62 años o más; también, llama la atención el que aparezca el dengue dentro de las primeras 10 causas de ingreso a las unidades de cuidado intensivo; sin embargo, ningún paciente que ingresó por esta patología desarrolló insuficiencia renal aguda.

Dentro de los factores de riesgo estudiados, la hipertensión arterial fue el antecedente patológico con mayor frecuencia, pero no se encontró ninguna asociación estadística significativa entre estos eventos. La sepsis fue el diagnóstico con mayor frecuencia entre los pacientes con IRA con un $8,6 \%$, seguido de la insuficiencia cardíaca aguda con un 5,4\%, lo que hace referencia a un grupo de enfermedades como: insuficiencia cardíaca congestiva, insuficiencia cardíaca descompensada y edema pulmonar; le siguen los eventos coronarios agudos en un 4,3\%. Para Uchino et al. (20) quienes realizaron un estudio multicéntrico en 23 países, entre 2001 y 2002, el factor más común que contribuyó a la falla renal aguda fue el shock séptico en un 47,5 \% y falla cardíaca como factor concomitante.

En el presente estudio se demostró que los pacientes que ingresaron a las UCI desarrollaron insuficiencia renal aguda, con una incidencia del 21,6\%; este resultado es superior al realizado por Uchino et al. (20), quienes concluyeron que el 5,7\% de los pacientes críticos ingresados durante el período de estudio presentaron IRA durante su estancia en la UCI. Sin embargo, estos resultados son inferiores a lo reportado por Abd ElHafeez et al. (21) para quienes el 37,4\% de los pacientes desarrollaron insuficiencia renal aguda después de 24 horas de ingreso en la UCI; también es inferior a lo encontrado por Fhurman et al. (22) quienes identificaron insuficiencia renal en el 52,6\% de los jóvenes entre 16 a 25 años que se encontraban en UCI; del mismo modo, lo evidenciaron Hoste et al. (23), al señalar que el 57,3 $\%$ (IC 95\% 55,0-59,6) de los pacientes presentaron esta complicación en UCI.

Al igual que en los casos anteriores, los resultados obtenidos por Capote et al. (24), presentan una incidencia de $22,4 \%$ con respecto a lo que ocurre en la ciudad de Bogotá, que según Moreno et al.(10) la suficiencia renal aguda en la unidad de cuidados intensivos desde junio a diciembre de 2006 fue de $22 \%$. El presente estudio arrojó una tasa de incidencia de insuficiencia renal aguda de 29,2 por cada 100 pacientes en un año de exposición. No hubo diferencias significativas en la incidencia de IRA entre el Hospital y la Clínica, siendo de 22,2 \% y 21,9 \% respectivamente.

Otros estudios han investigado sobre la incidencia de insuficiencia renal aguda, como es el caso de Cardoso et al. (25), quienes midieron la insuficiencia renal aguda en una unidad de cuidado intensivo pediátrico, asociándola con la duración de la estancia en la unidad de cuidados intensivos, la duración de la ventilación mecánica y la muerte hospitalaria $(\mathrm{p}<0,01)$; se debe precisar que en este grupo de pacientes ocurrió en 12,3\% durante el primer día posoperatorio. Por su parte, Caravaca-Fotan et al. (26) estudiaron el infarto agudo del riñón, encontrando que el 51,6\% de los pacientes incluidos en el estudio presentaron esta entidad de causas no cardíacas, siendo más jóvenes y con menos comorbilidad.

Teniendo en cuenta que este estudio fue realizado en dos instituciones de la ciudad, se encontraron diferencias significativas en los diagnósticos de los pacientes una vez presentaron IRA; así, para la Clínica el principal diagnóstico de los pacientes que desarrollaron IRA fue Insuficiencia cardíaca aguda 24,3 \% y en el Hospital la sepsis representando un $22 \%$; sin embargo, llama la atención que todos los pacientes con insuficiencia cardíaca aguda que desarrollaron insuficiencia renal aguda los aportó la Clínica. Esto concuerda con la fisiología de la sepsis y falla renal aguda $(27,28)$.

Con este estudio se prueba que el tiempo trascurrido entre el ingreso a la unidad de cuidado intensivo y el desarrollo de insuficiencia renal aguda, es el resultado de un análisis de supervivencia, en donde se demostró que el promedio en días en ocurrir el evento fue de 3,1 (IC 95 $\%$ 2,48-3,74). También, se verificó que en los pacientes con diagnósticos de posoperatorios, insuficiencia cardíaca aguda y trauma craneoencefálico presentaron IRA a los 9,82 (IC 95\% 8,42-11,2), 6,84 (IC $95 \%$ 4,21-9,48) y 4,5 (IC $95 \%$ 1,56-7,44) días respectivamente.

Del mismo modo, se midieron las intervenciones farmacológicas descritas en estudios anteriores que están relacionados con el desarrollo de la insuficiencia renal aguda, encontrándose que la nitroglicerina con un 47 $\%$ y la noradrenalina en un $43,2 \%$ fueron los medicamentos a los que los pacientes que desarrollaron IRA estuvieron más expuestos; por otro lado, los medios de contraste y la Vancomicina también se analizaron pero sus frecuencias fueron menores, lo que difiere con McCullough (29), quien menciona que los medios de con- 
ISSN-PRINT

1794-9831

E-ISSN 2322-7028

Vol. 16 No. 1

Ene - Abr 2019

Cúcuta, Colombia traste son responsables de un número significativo de casos de insuficiencia renal adquirida en el hospital, con efectos adversos sobre el pronóstico y los costos de atención médica.

No obstante, solo se encontró significancia estadística en el desarrollo de la insuficiencia renal aguda y la administración de nitroglicerina, en donde el promedio de días de aparición del evento fue en 6,7 días. Debe señalarse, que en este estudio no se evaluaron otros medicamentos identificados por Iavecchia et al. (13), quienes encontraron una incidencia de insuficiencia renal aguda durante la hospitalización de 9,6 por 1000 ingresos, en el $54,1 \%$ de los casos, en donde la insuficiencia renal aguda estaba relacionada con las drogas, siendo los fármacos más frecuentemente utilizados: los diuréticos, los agentes que actúan sobre el sistema renina-angiotensina, los inmunosupresores, los bloqueadores $\beta$, los bloqueadores de los canales de calcio, los medios de contraste y los antiinflamatorios no esteroideos.

Dentro de las limitaciones se encuentra el no disponer de fuentes de información secundaria; por otra parte, la historia clínica se convierte en un elemento vital para la investigación y el adecuado registro es la base para la validez de los datos, aunque la disposición de la historia clínica en físico generó al grupo investigador dificultades en el acceso.

Por otra parte, llama la atención el hecho de que más del $50 \%$ de la población que ingresa a las unidades de cuidado intensivo presenta la función renal alterada definida con valores de creatinina fuera de los límites establecidos para cada laboratorio, que en el caso de la Clínica sus rangos se definen entre 0,6 a $1,2 \mathrm{mg} / \mathrm{dl}$ y para el Hospital entre 0,5 a $1,0 \mathrm{mg} / \mathrm{dl}$ un rango más pequeño. Resultados que estás relacionados con la no inclusión de cerca del $45 \%$ de los pacientes al estudio, puesto que de los 339 pacientes solo se incluyeron 186, lo que se convirtió en limitación al estudiar un grupo reducido de pacientes.

En resumen, se puede indicar que el problema de salud pública que tiene el departamento del Tolima y Colombia, tal como lo presenta la Cuenta de alto costo (30) relacionada con las enfermedades crónicas no transmisibles, especialmente la enfermedad renal crónica, amerita la necesidad de ofrecer servicios de salud efectivos y eficientes; así como tener en cuenta la importancia de prevenir este tipo de enfermedad a través de estrategias y políticas efectivas.

\section{Conclusiones}

- La incidencia de insuficiencia renal aguda en pacientes hospitalizados en unidades de cuidados intensivos adultos fue del 21,6 \%. Por cada 100 pacientes por año de exposición solamente el 29,2 desarrollaron el evento y la incidencia de insuficiencia renal aguda en los pacientes del estudio fue de 21,6\% (IC $95 \%$ 17-30). La creatinina en promedio presentó elevaciones de $0,47 \mathrm{mg} /$ dl y en el gasto urinario mostró una disminución de $0,4 \mathrm{cc} / \mathrm{kg} / \mathrm{h}$ en los pacientes hospitalizados en unidades de cuidado intensivos adultos.

- El promedio de tiempo de seguimiento a los pacientes fue de 4,4 días, con una desviación estándar de 5,3 días; el tiempo promedio en desarrollar insuficiencia renal aguda en pacientes de las unidades de cuidado intensivo con diagnóstico de insuficiencia cardíaca aguda fue de 6,8 días (IC 95\% 4,21-9,48), el $50 \%$ presentaron el evento a los 6 días o menos y el $75 \%$ a los 4 días o menos.

- En cuanto a los pacientes con diagnóstico de posoperatorios, se encontró que el $75 \%$ presentaron insuficiencia renal aguda en 5 días o menos, con un promedio de 9,82 días (IC 95\% 8,42$11,2)$; en los que tenían diagnóstico de trauma craneoencefálico, se evidenció que el promedio en desarrollar la situación clínica estudiada fue de 4,5 días (IC $95 \%$ 1,56-7,44), de éstos el $50 \%$ presentaron insuficiencia renal aguda a los 3 días.

- La insuficiencia renal aguda hace parte de la historia natural de la enfermedad de la insuficiencia cardíaca aguda y los posoperatorios; sin embargo, en el caso del trauma craneoencefálico, este evento está relacionado con el manejo en la reanimación del trauma, por lo que las consecuencias para la práctica clínica incluyen mejorar la adherencia a las guías de manejo para evitar éste tipo de complicaciones.

- Para investigaciones futuras, se sugiere que se estudie a fondo a los pacientes con trauma craneoencefálico en los primeros momentos de la 
reanimación para determinar las causas de esta relación encontrada en el estudio. Así mismo, se recomienda aumentar el tiempo de observación entre seis meses a un año, realizar el seguimiento de manera prospectiva para tener mayor control del estudio, con el objetivo de proponer medidas para reducir la incidencia de insuficiencia renal aguda en la ciudad de Ibagué.

\section{Conflicto de Intereses}

Los autores declaran no tener ningún conflicto de interés

\section{Referencias Bibliográficas}

1. Monedero P, García Fernández N, Pérez Valdivieso JR, Vives M, Lavilla J. Insuficiencia renal aguda. Rev Esp Anestesiol Reanim. 2011; 58(6):365-74.

2. Atehortua L. Primer consenso colombiano en lesión renal aguda. Acta Colomb Cuid Intensivo. [Internet]. 2011 [consultado 25 de febrero de 2018]; 24(3). Disponible en: http://congresoatomico.com/ amci/images/documentos/SuplementoNOV2011_4.pdf

3. Higgins LB, Beltrán YH, Martínez GA. Calidad de vida relacionada con la salud en pacientes con enfermedad renal crónica. Rev Fac Med. 2015; 63(4):641-7.

4. Acuña L, Sánchez P, Soler LA, Alvis LF. Enfermedad renal en Colombia: prioridad para la gestión de riesgo. Rev Panam Salud Pública. 2016; 40:16-22.

5. Guerra Guerrero V, Sanhueza Alvarado O, Cáceres Espina M. Quality of life in people with chronic hemodialysis: association with sociodemographic, medical-clinical and laboratory variables. Rev Lat Am Enfermagem. 2012; 20(5):838-46

6. Medina MML. La enfermedad renal crónica en Colombia: necesidades en salud y respuesta del Sistema General de Seguridad Social en Salud. Gerenc Políticas Salud [Internet]. 2016 [citado 25 de febrero de 2018]; 15(30):212-233. Disponible en: http://revistas.javeriana.edu.co/index.php/gerepolsal/ article/view/17165

7. López Soracipa VA, España Barrios EA, Hernández Garcés AE. Estimación de calidad del cuidado de enfermería en pacientes sometidos a hemodiálisis. Rev Cienc Cuid. 2018; 15(1):71-82.

8. Zhou J, Yang L, Zhang K, Liu Y, Fu P. Risk factors for the prognosis of acute kidney injury under the Acute Kidney Injury Network definition: a retrospective, multicenter study in critically ill patients. Nephrol Carlton Vic. mayo de 2012; 17(4):330-7.

9. Capote Leyva E, Capote Pereira L, Castañer Moreno J, González M, Roberto S, et al. Letalidad asociada con la insuficiencia renal aguda en una unidad de cuidados intensivos de adultos. Rev Cuba Med Mil. 2008; 37(2).

10. Moreno A, Insuasty MI, Londoño D, D’Achiardi R, Garcia P. Clinical characteristics of the intensive care patients at the Hospital Universitario de San Ignacio with acute renal failure and factors associated with mortality. Acta Medica Colomb. 2011; 36(4):168-72.

11. Khwaja A. KDIGO Clinical Practice Guidelines for Acute Kidney Injury. Nephron Clin Pract. 2012; 120(4):c179-84.

12. Cortés JA, Soto R, Álvarez CA, Buitrago G, Camargo RD, Cataño JC, et al. Consensus for antimicrobial use in critically ill patients with renal failure or at risk of suffering it. Infectio. 2011;15(1):49-63.

13. Iavecchia L, Cereza García G, Sabaté Gallego M, Vidal Guitart X, Ramos Terrades N, de la Torre J, et al. Drug-related acute renal failure in hospitalised patients. Nefrol Publicacion Of Soc Española Nefrol. 2015; 35(6):523-32.

14. Oliveira JFP, Silva CA, Barbieri CD, Oliveira GM, Zanetta DMT, Burdmann EA. Prevalence and Risk Factors for Aminoglycoside Nephrotoxicity in Intensive Care Units. Antimicrob Agents Chemother. 2009;5 3(7):2887-91. 
ISSN-PRINT

$1794-9831$

E-ISSN 2322-7028

Vol. 16 No. 1

Ene - Abr 2019

Cúcuta, Colombia

15. Vallabhajosyula S, Sakhuja A, Geske JB, Kumar M, Poterucha JT, Kashyap R, et al. Role of Admission Troponin-T and Serial Troponin-T Testing in Predicting Outcomes in Severe Sepsis and Septic Shock. J Am Heart Assoc Cardiovasc Cerebrovasc Dis [Internet]. 2017 [citado 16 de marzo de 2018]; 6(9). Disponible en: https://www.ncbi.nlm.nih.gov/pmc/articles/PMC5634261/

16. Kellum JA. Acute kidney injury. Crit Care Med. abril de 2008; 36(4 Suppl): S141-145.

17. Kashani K, Al Khafaji A, Ardiles T, Artigas A, Bagshaw SM, Bell M, et al. Discovery and validation of cell cycle arrest biomarkers in human acute kidney injury. Crit Care. 2013;17(1):R25

18. Mehta RL, Kellum JA, Shah SV, Molitoris BA, Ronco C, Warnock DG, et al. Acute Kidney Injury Network: report of an initiative to improve outcomes in acute kidney injury. Crit Care. 2007;11(2):R31

19. Gobernación del Tolima - Secretaria de salud del Tolima. Análisis de Situación de Salud del Tolima con el Modelo de los Determinantes Sociales de Salud [Internet]. [citado 26 de febrero de 2018]. Disponible en: http://www.saludtolima.gov.co/wp-content/uploads/2014/08/ASIS-TOLIMA-2013.pdf

20. Uchino S, Kellum JA, Bellomo R, Doig GS, Morimatsu H, Morgera S, et al. Acute renal failure in critically ill patients: a multinational, multicenter study. JAMA J Am Med Assoc. 2005; 294(7):813-8.

21. Abd ElHafeez S, Tripepi G, Quinn R, Naga Y, Abdelmonem S, AbdelHady M, et al. Risk, Predictors, and Outcomes of Acute Kidney Injury in Patients Admitted to Intensive Care Units in Egypt. Sci Rep. 2017; 7(1):17163.

22. Fuhrman DY, Kane-Gill S, Goldstein SL, Priyanka P, Kellum JA. Acute kidney injury epidemiology, risk factors, and outcomes in critically ill patients 16-25 years of age treated in an adult intensive care unit. Ann Intensive Care. 2018; 8(1):26.

23. Hoste EAJ, Bagshaw SM, Bellomo R, Cely CM, Colman R, Cruz DN, et al. Epidemiology of acute kidney injury in critically ill patients: the multinational AKI-EPI study. Intensive Care Med. 2015; 41(8):1411-23.

24. Capote Pereira L, Elsa MB, Puga Torres M, Teuma Cortes I, Pozo Calderón M. Insuficiencia renal aguda en pacientes críticos ventilados: epidemiología y pronóstico a partir de la definición operativa de la acute kidney injury network: akin. Rev Cuba Med Intensiva Emerg. 2009; 9.

25. Cardoso B, Laranjo S, Gomes I, Freitas I, Trigo C, Fragata I, et al. [Acute kidney injury after pediatric cardiac surgery: risk factors and outcomes. Proposal for a predictive model]. Rev Port Cardiol Orgao Of Soc Port Cardiol Port J Cardiol Off J Port Soc Cardiol. febrero de 2016;35(2):99-104.

26. Caravaca-Fontán F, Pampa Saico S, Elías Triviño S, Galeano Álvarez C, Gomis Couto A, Pecharromán de las Heras I, et al. Acute renal infarction: Clinical characteristics and prognostic factors. Nefrol Publicacion Of Soc Espanola Nefrol. 2016; 36(2):141-8.

27. Regueira $T$, Andresen M, Mercado M, Downey P. Fisiopatología de la insuficiencia renal aguda durante la sepsis. Med Intensiva. octubre de 2011;35(7):424-32.

28. Trimarchi H, Nozieres C, Cámpolo Girard V, Lombi F, Smith C, Young P, et al. Injuria renal aguda en la sepsis grave. Med B Aires. junio de 2009; 69(3):321-6.

29. McCullough PA. Acute kidney injury with iodinated contrast. Crit Care Med. abril de 2008;36(4):S204

30. Cuenta de alto costo. Situación de la enfermedad renal crónica en Colombia. [Internet]. Fondo Colombiano de Enfermedades de Alto Costo; 2013. [consultado 23 de febrero de 2018]; 24(3). Disponible en: https://www.minsalud.gov.co/sites/rid/Lists/BibliotecaDigital/RIDE/INEC/CAC/SITUACION DE_LA_ENFERMEDAD_RENAL_CRONICA_2013.pdf 